\title{
Cardiac valve calcification: characteristics of patients with calcification of the mitral annulus or aortic valve
}

\author{
Arthur Boon, Emile Cheriex, Jan Lodder, Fons Kessels
}

St Anna Hospital, Geldrop, Netherlands A Boon

Department of Cardiology, University Hospital Maastricht,

Netherlands

E Cheriex

Department of Neurology, University Hospital Maastricht, Netherlands

J Lodder

Department of Epidemiology,

University of Limburg, Maastricht,

Netherlands

F Kessels

Correspondence to:

Dr Boon, Department of

Neurology of the St Anna

Hospital, PO Box 90, 5660

$\mathrm{AB}$, Geldrop, Netherlands.

Accepted for publication 15 August 1997

\begin{abstract}
Aims-To determine whether mitral annular calcification and aortic valve calcification, with or without stenosis, are expressions of atherosclerotic disease. Methods-The incidence of atherosclerotic risk factors was analysed in patients with mitral annular calcification and aortic valve calcification and in control patients from a prospective echocardiographic database of 8160 consecutive patients; 657 patients $(8 \%)$ were identified with mitral annular calcification and 815 (9\%) with a calcified aortic valve, of whom $515(6.3 \%)$ had stenosis with a minimal aortic valve gradient of $16 \mathrm{~mm} \mathrm{Hg}$. In these patients, cardiac and vascular risk factors were compared with 568 control patients using multiple logistic regression analysis.
\end{abstract}

Results-Age (odds ratio (OR) varying from 5.78 to 104, depending on age class), female sex (OR 1.75), hypertension (OR 2.38), diabetes mellitus (OR 2.85), and hypercholesterolaemia (OR 2.95) were strongly and significantly associated with aortic valve calcification without stenosis, as were age (OR varying from 8.82 to 67 , depending on age class), female sex (OR 2.22), hypertension (OR 2.72), diabetes mellitus (OR 2.49), and hypercholesterolaemia (OR 2.86) with mitral annular calcification. Age (OR varying from 1.11 to 7.7), hypertension (OR 1.91), and hypercholesterolaemia (OR 2.55) were strongly and significantly associated with stenotic aortic valve calcification.

Conclusions-Mitral annular calcification and stenotic or non-stenotic aortic valve calcification have a high incidence of atherosclerotic risk factors, suggesting they should be considered as manifestations of generalised atherosclerosis. (Heart 1997;78:472-474)

Keywords: mitral annular calcification; aortic valve calcification; calcified aortic stenosis; risk factors

Mitral annulus calcification, aortic valve calcification, and calcific aortic valve stenosis represent a degenerative process that occurs mainly in the elderly. Calcific deposits lead to cardiac conduction disturbances in these patients, ${ }^{12}$ or to embolism due to dislodgement of calcified material in the blood stream. The degenerative mechanism may accelerate when mechanical stress on the valvar apparatus is increased in hypertension and congenital or acquired valvar disorders. Prevalence of mitral annulus calcification and aortic valve calcification increases with age and often the conditions coexist. ${ }^{3}$ Therefore the question arises as to whether the valvar calcifications are the result of increased valvar stress (as is the case in hypertension), an expression of more generalised atherosclerosis, or reflect a primary degenerative process, progressing with advancing age. Among the reports that suggest an association of mitral annulus and aortic valve calcification with cardiac and vascular risk factors is one population based study of 162 patients with mitral annulus calcification, which describes a multiple logistic regression model containing clinical risk factors. ${ }^{4}$ We analysed whether there is a significant association of calcification of the mitral annulus or aortic valve with specific clinical and echocardiographic risk factors for generalised atherosclerotic disease.

\section{Methods}

The study population was recruited from a specially designed prospective echocardiographic database of 8160 consecutive patients examined between 1 January 1985 and 1 January 1990 at the department of cardiology of the University Hospital of Maastricht. The purpose and design of this registry is described elsewhere. ${ }^{5}$ All patients with mitral annulus calcification or aortic valve calcification were selected for analysis of concomitant vascular risk factors. Risk factors were also studied in the control group, which was a randomly selected sample of 562 patients from the remaining patients without aortic valve or mitral annular calcification in the same database. Study and control patients were consecutive ambulatory or inpatients referred for echocardiography. Indications for echocardiography did not differ between patients and controls. The University Hospital is the only hospital in the region. Therefore indications for referral to the hospital, as well as those for echocardiography, were representative of a university cardiology practice. In none of the patients or controls was suspicion of mitral annulus calcification or aortic valve calcification a reason for echocardiography, or for referral to the hospital. The following risk factors were recorded: age, sex, hypertension (known hypertension treated with antihypertensive drugs, two or more blood pressure recordings greater than $160 / 90 \mathrm{~mm} \mathrm{Hg}$ ), 
Table 1 Baseline characteristics

\begin{tabular}{lllll}
\hline & MAC (n=657) & AVC (n=300) & CAVS (n=515) & Controls (n=562) \\
\hline Mean age (SD) [median] (years) & $72(10)[74]$ & $74(10)[75]$ & $67(15)[71]$ & $53(18)[58]$ \\
Range (years) & 21 to 96 & 21 to 92 & 7 to 96 & 0 to 90 \\
Age <45 years & 8 & 4 & 42 & 163 \\
Age 45 to 54 years & 26 & 6 & 30 & 86 \\
Age 55 to 64 years & 97 & 35 & 101 & 132 \\
Age $\geqslant 65$ years & 526 & 255 & 342 & 181 \\
Men/women & $220(33) / 437(67)$ & $106(35) / 194(63)$ & $242(46) / 273(54)$ & $338(60) / 224(40)$ \\
Hypertension & $290(44)$ & $136(45)$ & $154(30)$ & $96(17)$ \\
Ischaemic heart disease & $382(50)$ & $179(59)$ & $249(48)$ & $225(40)$ \\
Diabetes mellitus & $124(19)$ & $68(23)$ & $51(10)$ & $43(8)$ \\
Hypercholesterolaemia & $36(5)$ & $18(6)$ & $22(4)$ & 5.9 \\
Mean serum cholesterol (mmol/1) & 6.6 & 6.5 & 6.6 & $28(5)$ \\
Peripheral arteriosclerosis & $55(8)$ & $14(10)$ & $36(7)$ & $30(5)$ \\
Atrial fibrillation & $32(4)$ & $6(2)$ & $59(11)$ & $19(3)$ \\
Mitral stenosis & $18(2)$ & $95(31)$ & $271(52)$ & $218(39)$ \\
Mitral regurgitation & $220(33)$ & $0 / 0$ & $2 / 19$ & $26 / 39$ \\
Aortic/mitral valve prosthesis & $6 / 0$ & $26(9)$ & $61(12)$ & $60(11)$ \\
Coronary artery bypass & $67(10)$ & & &
\end{tabular}

Values are number (\%) unless otherwise stated.

MAC, mitral annular calcification; AVC, aortic valve calcification without stenosis; CAVS, stenotic aortic valve calcification.

diabetes mellitus (known diabetes treated with diet or drugs or both; or either a fasting serum glucose of more than $7 \mathrm{mmol} / \mathrm{l}$, or a postprandial serum glucose of more than $11 \mathrm{mmol} / 1$ measured on at least two separate occasions), ischaemic heart disease (myocardial infarction, angina pectoris), coronary artery bypass grafting, oral anticoagulants or salicylates, previous stroke or transient ischaemic attacks, serum cholesterol (the mean of all available measurements with or without treatment with cholesterol lowering drugs), hypercholesterolaemia (known treated hypercholesterolaemia or fasting or non-fasting serum cholesterol concentrations higher than $6.5 \mathrm{mmol} / \mathrm{l}$ ), symptomatic peripheral arteriosclerotic disease (that is, intermittent claudication), and atrial fibrillation. For convenience, preventive measures are also considered as risk factors in the text and tables. Among other factors, the following echocardiographic variables were recorded: aortic valve calcification (defined as bright dense echoes of $\geqslant 1 \mathrm{~mm}$ size on one or more cusps and decreased mobility of the involved cusp; if Doppler velocity was less than $2 \mathrm{~m} / \mathrm{s}$, measured with continuous Doppler, flow was considered to be within normal limits); calcific aortic valve stenosis (defined as having a maximum pressure gradient exceeding $16 \mathrm{~mm} \mathrm{Hg}$ ); mitral annulus calcification (defined as bright echoes in mitral annulus on two dimensional echocardiogram with "stone shadow"); mitral stenosis (defined as rheumatic mitral stenosis with increased velocities over the valve and a mitral valve area $\leqslant 2.5 \mathrm{~cm}^{2}$, or non-rheumatic valvar disease if mitral annulus calcification and fibrosis caused a more than physiological gradient and a mitral valve orifice $\leqslant 2.5 \mathrm{~cm}^{2}$ ); mitral regurgitation; enlarged left atrium (diameter $45 \mathrm{~mm}$ or more); cardiac valve prosthesis. The rationale for including these variables in the study model was to search for associations between calcified cardiac valves and risk factors for atherosclerosis, or other cardiac findings suggested in previous studies.

\section{STATISTICS}

Statistical analysis was done with stepwise multiple logistic regression analysis with mitral annulus calcification, aortic valve calcification, and calcified aortic valve with stenosis, respectively, as dependent variables, and the control group as reference. The clinical and echocardiographic variables listed above could be entered into the model when the $\mathrm{p}$ level of entrance was 0.10 or lower.

\section{Results}

Six hundred and fifty seven patients had mitral annulus calcification, and 815 had aortic valve calcification, leading to calcific aortic valve stenosis in 515 . Of the patients with mitral annulus calcification, 289 had aortic valve calcification and 113 had calcific aortic valve stenosis. Baseline characteristics of the groups are shown in table 1. Logistic regression analysis on risk factors is shown in table 2. Age, hypertension, and an enlarged left atrium were

Table 2 Multiple logistic regression analysis of the risk factors associated with mitral annular calcification (MAC) and aortic valve calcification without $(A V C)$ or with (CAVS) stenosis, compared with controls

\begin{tabular}{|c|c|c|c|c|c|c|}
\hline \multirow[b]{2}{*}{ Risk factors } & \multicolumn{2}{|l|}{$M A C$} & \multicolumn{2}{|l|}{$A V C$} & \multicolumn{2}{|l|}{$C A V S$} \\
\hline & $O R(95 \% C I)$ & $p$ & OR $(95 \% C I)$ & $p$ & OR $(95 \% C I)$ & $p$ \\
\hline Age $45-55 /<45$ years & $8.82(3.37$ to 23.1$)$ & 0.0 & $5.78(1.13$ to 30.6$)$ & 0.03 & $1.11(0.56$ to 2.14$)$ & 0.7 \\
\hline Age $55-65 /<45$ years & $18.6(7.54$ to 45$)$ & 0.0 & 23.3 (5.33 to 102$)$ & 0.0 & $2.28(1.30$ to 4.0$)$ & 0.0 \\
\hline Age $>65 /<45$ years & $67(27$ to 161$)$ & 0.0 & $104(24.6$ to 440$)$ & 0.0 & 7.7 (4.68 to 12.7$)$ & 0.0 \\
\hline Men/women & $0.45(0.33$ to 0.63$)$ & 0.0 & $0.57(0.38$ to 0.68$)$ & 0.0 & & \\
\hline Hypertension & $2.72(1.92$ to 3.86$)$ & 0.0 & $2.38(1.55$ to 3.65$)$ & 0.0 & $1.91(1.31$ to 2.78$)$ & 0.0 \\
\hline Diabetes mellitus & $2.49(1.4$ to 4.14$)$ & 0.0 & $2.85(1.58$ to 5.13$)$ & 0.0 & & \\
\hline Hypercholesterolaemia & $2.86(1.16$ to 7.08$)$ & 0.02 & $2.95(0.99$ to 8.82$)$ & 0.05 & $2.55(0.90$ to 7.17$)$ & 0.07 \\
\hline Anterior myocardial infarct & $0.65(0.41$ to 1.03$)$ & 0.06 & $0.47(0.26$ to 0.85$)$ & 0.01 & $0.18(0.10$ to 0.34$)$ & 0.0 \\
\hline Atrial fibrillation & & & $0.41(0.16$ to 1.02$)$ & 0.05 & & \\
\hline Mitral regurgitation & $0.45(0.32$ to 0.63$)$ & 0.0 & $0.48(0.31$ to 0.74$)$ & 0.0 & & \\
\hline Rheumatic mitral stenosis & & & & & $2.29(1.11$ to 4.74$)$ & 0.02 \\
\hline
\end{tabular}

OR, adjusted odds ratio; $\mathrm{CI}$, confidence interval. 
strongly associated with mitral annulus calcification, aortic valve calcification, and calcific aortic valve stenosis. Hypercholesterolaemia was associated with each of the valvar calcifications, although significantly only with mitral annulus calcification. Female sex and diabetes mellitus were significantly associated with aortic valve calcification and mitral annulus calcification, but not with calcific aortic valve stenosis. Probably because both patients and controls were referred to cardiologists, some conditions were negatively associated with the valve calcifications: echocardiographic anterior wall infarction with aortic valve calcification and mitral annular calcification, and atrial fibrillation with aortic valve calcification. Although due to cardiology referral the a priori chance of having cardiovascular disease was high in both patients and controls, vascular risk factors were significantly associated with all three types of cardiac valve calcification in this study.

\section{Discussion}

Associated risk factors were, for mitral annulus calcification and aortic valve calcification: age, female sex, hypertension, diabetes mellitus, hypercholesterolaemia, and an enlarged left atrium; and for calcific aortic valve stenosis: age, hypertension, hypercholesterolaemia, and an enlarged left atrium.

\section{MITRAL ANNULAR CALCIFICATION}

A study of mitral annulus calcification ${ }^{4}$ showed an association with age, female sex, hypertension, and an enlarged left atrium, and on univariate analysis also with atrial fibrillation and aortic stenosis. We found a high prevalence of aortic valve calcification (43\%) and calcific aortic valve stenosis $(17 \%)$ in patients with mitral annulus calcification. However, there was no significant association between aortic valve calcification, calcific aortic valve stenosis, and mitral annulus calcification after adjusting for risk factors, and therefore this concomitance may be completely explained by common vascular risk factors in patients with these conditions. Atrial fibrillation was more frequent in our control group than in that of the population based Framingham study, which may explain why we did not find an association between mitral annulus calcification and atrial fibrillation. However, the higher percentage of atrial fibrillation among patients with mitral annulus calcification in the latter study may represent a higher incidence of cardiovascular risk factors, especially enlarged left atrium, and possibly age, which were not adjusted for in the analysis. ${ }^{4}$ Differences between the Framingham study and our own study in the association of mitral annulus calcification with diabetes mellitus and hypercholesterolaemia may be explained by our definitions, which resulted in treated patients being considered as having the condition. Six other studies have reported cardiovascular risk factor profiles in patients with mitral annulus calcification, but using univariate models in age and sex matched patients, not adjusted for concomitant vascular risk factors. ${ }^{6-11}$ Why mitral annulus calcification prevails in women is unclear, although a role of increased serum parathyroid hormone resulting in hypovitaminosis D has been proposed. ${ }^{12}$

\section{AORTIC VALVE CALCIFICATION}

Epidemiological data about risk factors for aortic valve calcification are not available. Patients with calcific aortic valve stenosis more often had angina pectoris, hypertension, or sudden cardiac death, ${ }^{13}$ or cardiac conduction disorders. ${ }^{12}$ Our findings are in agreement with Roberts, ${ }^{314}$ who stated that factors predisposing to atherosclerosis also predispose to aortic valve calcification.

\section{CONCLUSION}

We have shown that mitral annular and aortic valve calcification have similar cardiac and vascular risk factors and should be regarded as comparable expressions of underlying age related cardiac manifestations of atherosclerosis.

1 Nair CK, Sketch MH, Ahmed I, Thomson W, Ryschon K, Woodruff MP, et al. Calcific valvular aortic stenosis with and without mitral annular calcium. Am $f$ Cardiol and without mitra

2 Nair CK, Aronow WS, Stokke K, Mohiuddin SM, Thomson W, Sketch MH. Cardiac conduction defects in patients older than 60 years with aortic stenosis with and without mitral anular calcium. Am f Cardiol 1984;53:16972

3 Roberts WC. Morphologic features of the normal and abnormal mitral valve. Am f Cardiol 1983;51:1005-28.

4 Savage D, Garrison RJ, Castelli WP, McNamara PM, Anderson SJ, Kannel WB, et al. Prevalence of submitral (annular) calcium and its correlates in a general population-based sample (the Framingham study). Am $\mathcal{f}$ Cardiol 1983;51:1375-8.

5 Mulleneers R, Cheriex E, Dassen W, Bleijlevens B, Wellens H. CAESARS: the cardiac storage and retrieval network system. Comput Cardiol 1990:211-14.

6 Nair CK, Sudhakaran C, Aronow WS, Thomson W, Woodruff MP, Sketch MH. Clinical characteristics of patients younger than 60 years with mitral anular calcium: comparison with age- and sex-matched control subjects. Am ₹ Cardiol 1984;54:1286-7.

7 Aronow WS, Schwartz KS, Koenigsberg M. Correlation of atrial fibrillation with presence or absence of mitral anular calcium in 604 persons older than 60 years. Am $\mathcal{F}$ Cardiol 1987;59:1213-14.

8 Nair CK, Thomson W, Ryschon K, Cook C, Hee TT, Sketch MH. Long-term follow-up of patients with echocardiographically detected mitral anular calcium and comparison with age- and sex-matched control subjects. Am ₹ Cardiol 1989;63:465-70.

9 Aronow WS, Koenigsberg M, Kronzon I, Gutstein H. Association of mitral anular calcium with new thromboembolic stroke and cardiac events at 39-month follow-up in elderly patients. Am f Cardiol 1990;65:1511-12.

10 Benjamin EJ, Plehn JF, D’Agostino RB, Belanger AJ, Comai $\mathrm{K}$, Fuller DL, et al. Mitral annular calcification and the risk of stroke in an elderly cohort. N Engl F Med 1992;327:374-

11 Nair CK, Aronow WS, Sketch MH, Mohinddin SM, Pagano T, Esterbrooks DJ, et al. Clinical and echocardiographic characteristics of patients with mitral anular calcification. Comparison with age- and sex-matched control subjects. Am f Cardiol 1983;51:992-5.

12 Shiraki M, Miyagawa A, Akiguchi I, Ito H, Ohkawa S, Sugiura $M$. Evidence of hypovitaminosis $D$ in patients with mitral ring calcification. $\not$ pp $n$ Heart $\mathcal{f}$ 1988;29:801-8.

13 Wren C, Petch MC. Calcific aortic stenosis. $\mathcal{F} R$ Coll Physicians Lond 1983;17:192-5.

14 Roberts WC. The senile cardiac calcification syndrome. Am f Cardiol 1986;58:572-4. 